\title{
Improving deformable image registration with point metric and masking technique for postoperative breast cancer radiotherapy
}

\author{
Xin Xie ${ }^{1}$, Yuchun Song ${ }^{1}$, Feng Ye ${ }^{2}$, Hui Yan ${ }^{1}$, Shulian Wang ${ }^{1}$, Xinming Zhao $^{2}$, Jianrong Dai $^{1}$ \\ ${ }^{1}$ Department of Radiation Oncology, ${ }^{2}$ Department of Diagnostic Radiology, National Cancer Center/National Clinical Research Center for Cancer/ \\ Cancer Hospital, Chinese Academy of Medical Sciences and Peking Union Medical College, Beijing, China
}

Correspondence to: Jianrong Dai, PhD; Hui Yan, PhD. Department of Radiation Oncology, National Cancer Center/National Clinical Research Center for Cancer/Cancer Hospital, Chinese Academy of Medical Sciences and Peking Union Medical College, Beijing 100021, China. Email: dai_jianrong@163.com; hui.yan@cicams.ac.cn.

\begin{abstract}
Background: Deformable image registration (DIR) is increasingly used for target volume definition in radiotherapy. However, this method is challenging for postoperative breast cancer patients due to the large deformations and non-correspondence caused by tumor resection and clip insertion. In this study, an improved B-splines based DIR method was developed to address this issue for higher registration accuracy.

Methods: The conventional B-splines based DIR method was improved with the introduction of point metric and masking technique. The point metric minimizes the distance between 2 point sets with known correspondence for regularization of intensity-based B-splines registration. The masking technique reduces the influence of non-corresponding regions in breast computed tomography (CT) images. Two sets of CT images before and after breast surgery were used for image registration. One set was the diagnostic CT image acquired before surgery, and another set was the planning CT image acquired after surgery for breast cancer radiotherapy. A total of 26 sets of CT images from 13 patients were collected retrospectively for the test. The improved DIR method's registration accuracy was evaluated by target registration error (TRE), the Jacobian determinant, and visual assessment.

Results: For soft tissue, the difference in the median TRE between the improved DIR method and the conventional DIR method was statistically significant $(2.27$ vs. 5.88, $\mathrm{P}<0.05)$. The Jacobian determinant of the deformation field was positive for all patients. For visual assessment, the improved DIR method with point metric achieved better matching for soft tissue.

Conclusions: The improved DIR method's registration accuracy was higher than the conventional DIR method based on the preliminary results. With point metric and masking technique, the influence of large deformations and non-correspondence on registration between pre- and post-operative CT images can be effectively reduced. Therefore, this method provides a feasible way for target volume definition in postoperative breast cancer radiotherapy treatment planning.
\end{abstract}

Keywords: B-splines; deformable image registration (DIR); postoperative breast cancer radiotherapy

Submitted May 27, 2020. Accepted for publication Oct 23, 2020.

doi: $10.21037 /$ qims-20-705

View this article at: http://dx.doi.org/10.21037/qims-20-705

\section{Introduction}

Breast cancer is the most commonly diagnosed cancer and the leading cause of cancer death among women worldwide (1). Breast-conserving therapy (BCT), which involves a wide local excision followed by radiotherapy to the whole breast, has become the standard treatment for early-stage breast cancer (2). The addition of boost irradiation to the tumor bed after whole breast irradiation (WBI) has shown further improvement of local control (3). As most local recurrences appear close to the tumorectomy 
cavity, accelerated partial breast irradiation (APBI), which only irradiates the tumor bed and its surrounding breast tissue, has become an alternative to WBI for patients with low risk of recurrence (4). For either irradiation mode, accurate delineation of the breast tumor bed and its target volume is essential. However, the target volume varies widely in terms of specimen volume, seroma size, clarity, surgical clips, inter-observer variability, and other factors (5).

Registering the computed tomography (CT) image acquired before surgery to the CT image acquired after surgery can help define the target volumes for radiotherapy treatment planning. However, considering soft tissue nature, the image registration for breast is difficult (6). Breast is a non-rigid object which undergoes large and complex deformations in shape and volume. Due to surgical operation and postoperative breast changes, it is difficult to match the pre- and post-operative CT scans using rigid image registration methods (7-9). Recently, deformable image registration (DIR) has been introduced to define the breast tumor bed with different imaging modalities, including CT (10), contrast CT (11), magnetic resonance imaging (MRI) (12) and positron emission tomography/ computed tomography (PET/CT) (13). In general, the registration accuracy of DIR methods is higher than that of rigid registration methods.

According to matching information used in registration (14), the DIR methods can be divided into two categories: feature-based DIR and intensity-based DIR. So far, there are no reports on feature-based DIR in breast cancer radiotherapy due to the difficulty in identifying landmarks in clinical practice. For intensity-based DIR, there are several applications. However, certain issues have to be considered, which usually do not exist in conventional registration applications $(15,16)$. First, for patients undergoing breast-conserving surgery, the tumor is resected, and the tumor bed displays different image intensities due to the insertion of surgical clips and postoperative changes (such as seromas). These intensity changes will compromise the accuracy of intensity-based DIR methods $(17,18)$. Second, the traditional evaluation metrics for image registration are not directly applicable (16). Since the tumor is resected, there is no direct correspondence between the tumor and its bed. Third, the final deformation field is discontinuous due to tumor resection and clip insertion. This discontinuity will cause an ill-conditioned and noninvertible transformation matrix. Fourth, not all breast tumors are visible on preoperative CT images, particularly for CT scans without contrast usage. The tumor volume on the preoperative CT image is different from the real tumor volume resected during surgery. Additionally, the uniform expansion of the preoperative tumor volume does not well represent the resected tumor volume due to asymmetric excision of the tumor (19).

There are several DIR methods adopted by commercial image registration software, such as MIM (20), Velocity (13), and Mirada (11), of which the performance of contour propagation has been thoroughly investigated (21). Although most studies have indicated that DIR is helpful in contour delineation, $\mathrm{Yu}$ et al. (12) found a relatively poor spatial overlap for both the whole breast and the target volumes. These studies focused mainly on commercial image registration software's clinical applications and less on the technical aspects of DIR methods. To investigate the reliability of the DIR methods, Wodzinski et al. (22) examined different variants of the Demons algorithm. The nonparametric Demons algorithms were compared with the parametric B-spline free-form deformations (FFD), which employed analytical gradient calculation (23) to speed up the registration process. For the initial alignment procedure, the iterative closest point algorithm was comparable with the intensity-based rigid registration (24). To further validate the deformation correctness, Wodzinski et al. (10) proposed alternative artificial deformations that model the tumor bed creation process. A comprehensive evaluation demonstrated that the symmetric Demons algorithm provides the most accurate soft tissue alignment, while B-splines FFD and TV-L1 optical flow are not appropriate. Although the dedicated artificial deformation models were proposed to mimic real soft tissue motions, the ideal solution to validate the deformation would be a method to incorporate with biomechanical analysis. Besides, the above studies concentrated mostly on the tumor bed rather than the clinical target volume (CTV). Since CTV is defined as the extension of the tumor bed, which includes more surrounding soft tissue areas, the DIR method could pay more attention to the alignment of surrounding soft tissue, rather than the local discontinuities near the tumor bed region.

In this study, the conventional B-splines based DIR method was improved with the introduction of point metric and masking technique to reduce the influence of discontinuities caused by tumor resection and clip insertion on registration accuracy. In Method Section, the procedure of the improved intensity-based DIR method is introduced. Then, the applications of the point metric and masking technique are explained in detail. Next, the experiments 


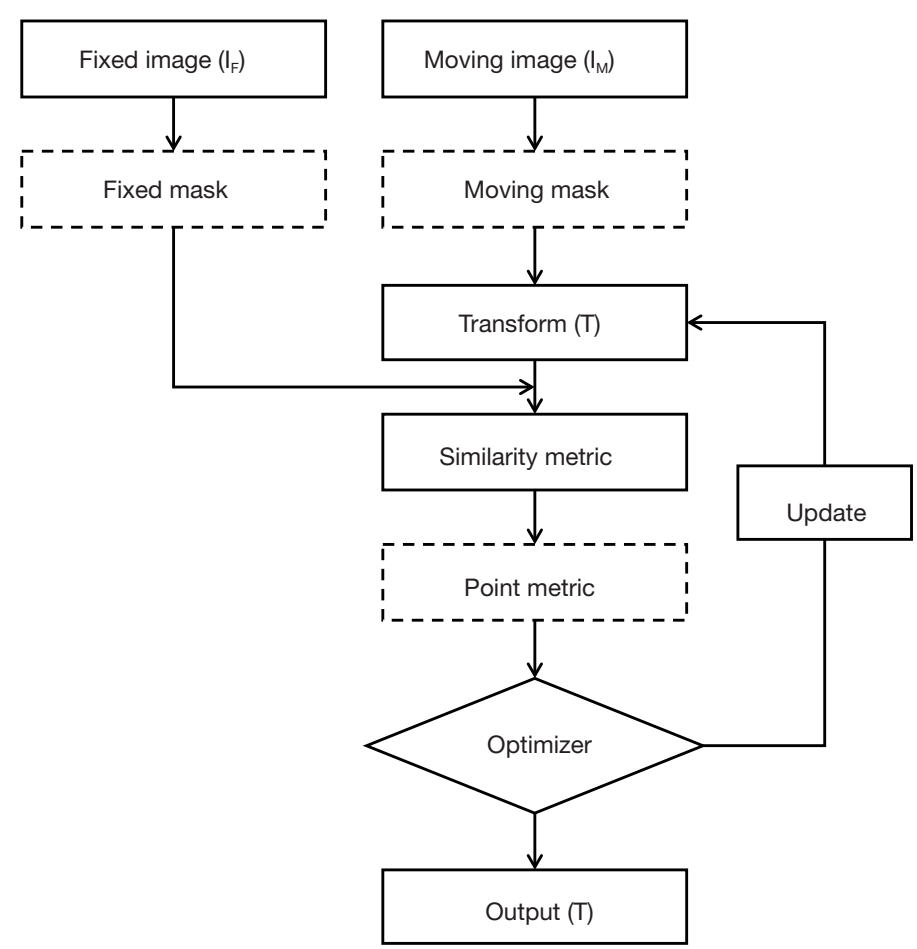

Figure 1 Flowchart of the improved image registration process. Solid boxes represent the conventional procedure, while dashed boxes represent the introduced components (point metric and masking technique).

and evaluation methods are described. In Results Section, the improved DIR method's performance combined with point metric and masking technique is reported. Finally, the proposed method's advantages and disadvantages are discussed, and future work is prospected.

\section{Methods}

\section{Improved intensity-based DIR}

The flowchart of the improved image registration process is illustrated in Figure 1. Image registration aims to find a transform $T$ that spatially aligns a moving image $I_{M}$ to a fixed image $I_{F}$. The masking technique is employed, and both masks for fixed and moving images are generated. For intensity-based image registration, the optimal alignment is generally determined by optimizing a similarity metric between image intensities. In this study, mutual information (MI) was used because it is appropriate for circumstances where corresponding anatomies' pixel intensities are inherently different. Additionally, a point metric was introduced to regularize the intensity-based registration. The registration was performed in two steps. First, the global registration, affine transformation (AT), was used for initial alignment between fixed and moving images. Then the local registration, B-splines based DIR (25), was used to refine the initial alignment between the two sets of images. In this study, an iterative stochastic gradient descent optimizer was employed, and its principles are described in Appendix 1.

\section{Point metric and masking technique}

A point metric (26) minimizes the distance between two point sets with known correspondence. It is defined as the mean Euclidean distance:

$$
\mathrm{S}_{\mathrm{CP}}=\frac{1}{\mathrm{P}} \sum_{\mathrm{x}_{\mathrm{F}_{\mathrm{i}}}}\left\|\mathrm{x}_{\mathrm{M}_{\mathrm{i}}}-\mathrm{T}_{\mu}\left(\mathrm{x}_{\mathrm{F}_{\mathrm{i}}}\right)\right\|
$$

where $P$ is the number of points $x_{i}, x_{F_{i}}$ and $x_{M_{i}}$ are corresponding points from the fixed and moving image point sets, respectively. We modeled the non-rigid transform $T$ with a $\mathrm{B}$-splines based deformation field, where $\mu$ is parameters of the transformation $T$. This metric was used to regularize an intensity-based registration by considering those positions where their correspondences were known. The derivative of $S_{C P}$ is: 


$$
\frac{\partial}{\partial \mu} \mathrm{S}_{\mathrm{CP}}=-\frac{1}{\mathrm{P}} \sum_{\mathrm{x}_{\mathrm{F}}} \frac{1}{\left\|\mathrm{x}_{\mathrm{M}_{\mathrm{i}}}-\mathrm{T}_{\mu}\left(\mathrm{x}_{\mathrm{F}_{\mathrm{i}}}\right)\right\|}\left(\mathrm{x}_{\mathrm{M}_{\mathrm{i}}}-\mathrm{T}_{\mu}\left(\mathrm{x}_{\mathrm{F}_{\mathrm{i}}}\right)\right) \frac{\partial \mathrm{T}}{\partial \mu}\left(\mathrm{x}_{\mathrm{F}_{\mathrm{i}}}\right)
$$

The corresponding point sets in each patient contained an average 30 pairs of points located in unaffected breast tissue and were marked manually by the physician.

The masking technique is mainly for setting the region of interest (ROI). Masks can be used for both the fixed and the moving images. A fixed image mask is sufficient to focus the registration on an ROI, since samples are drawn from the fixed image. A mask for the moving image excludes nonsense grey values since areas outside the mask do not actively contribute to the cost function. The mask is used for dilated whole breast subtracting $2 \mathrm{~cm}$ expansion of the tumor on the diagnostic CT (the fixed image). If the tumor expansion is not subtracted, it means no mask for the fixed image in this context. The mask is used for dilated whole breast subtracting obvious postoperative changes such as clips and seromas near the tumor bed region on the planning CT (the moving image). If postoperative changes are not subtracted, it means no mask for the moving image in this context.

For testing purposes, registration based on the AT and the conventional DIR method was performed to obtain a standard result. Examples of fixed and moving images in the conventional DIR method are shown in Figure $2 A, B$, respectively. Three combinations of point metric and masking technique on the effect of registration accuracy were investigated. The first combination (DIR-1) consisted of the fixed image (Figure 2C) and the moving image (Figure 2D) both without point metric but with masking technique. The second combination (DIR-2) consisted of the fixed image (Figure $2 E$ ) and the moving image (Figure $2 F$ ) both with point metric but without masking technique. The third combination (DIR-3) consisted of the fixed image (Figure 2G) and the moving image (Figure 2H) both with point metric and masking technique.

\section{Experiments}

The local ethics committee approved this study, and informed consent was waived in this retrospective study. The test data set consisted of 26 CT scans collected from 13 patients who underwent breast-conserving surgery and postoperative radiotherapy in our hospital. All tumors were clearly visible on the diagnostic CT scans, and their volumes varied from 630 to $5,420 \mathrm{~mm}^{3}$, averaging $1,621 \mathrm{~mm}^{3}$. The dimensions of CT volume varied from $512 \times 512 \times 57$ to
$512 \times 512 \times 86$ in voxels, and the slice thickness was $5.0 \mathrm{~mm}$. In-plane image resolution varied from $0.68 \times 0.68 \mathrm{~mm}$ to $1.37 \times 1.37 \mathrm{~mm}$. The registrations were performed on a 64 bit PC equipped with Intel Core i5 CPU 3.4-GHz and 32 GB RAM.

Elastix registration software developed by Klein and Staring $(27,28)$ (http://elastix.isi.uu.nl) was employed in this study. The AT initialized the intensity-based registration. A multi-resolution B-splines based registration was then performed. In the multi-resolution approach, Gaussian pyramid was used to smooth and down-sample the image at different scales. Here, we chose three resolutions. The grid size of the control points of the B-splines model was set to 16. Each scale's grid space was set to [4 2 1 1 ] times a physical unit (in $\mathrm{mm}$ ). The larger grid size allowed the registration to match large structures and skip small structures in the multi-resolution registration. The smaller grid size ensured that the detailed structures in the breast could be matched.

\section{Evaluations}

The methods' performances were evaluated by the standards of target registration error (TRE), the Jacobian determinant, and visual assessment.

TRE (29) is based on the Euclidean distance between corresponding points in the fixed and the moving images. Mean TRE value is defined as follows:

$$
\operatorname{TRE}_{\text {mean }}=\frac{1}{\mathrm{I}} \sum_{\mathrm{i}} \sqrt{\left(\mathrm{x}_{\mathrm{i}}-\mathrm{x}_{\mathrm{i}}^{\prime}\right)^{2}+\left(\mathrm{y}_{\mathrm{i}}-\mathrm{y}_{\mathrm{i}}^{\prime}\right)^{2}+\left(\mathrm{z}_{\mathrm{i}}-\mathrm{z}_{\mathrm{i}}^{\prime}\right)^{2}}
$$

where $\mathrm{i}$ is a single point indicator, I is the total number of points, $x, y, z$ denote the fixed point coordinates, and $x^{\prime}, y^{\prime}, z^{\prime}$ denote the corresponding point coordinates in the transformed moving image. It is noted that there is no direct correspondence between the tumor and its bed. We grouped all points into three categories according to their locations at unaffected soft tissues (within $5 \mathrm{~cm}$ away from tumor area), rigid structures (ribs, breastbone), and body boundaries (nipple) as illustrated in Figure 3. In total, 30 pairs of points were chosen for each patient. The median of the registration error, together with 25 and 75 percentile values were reported. The BenjaminiHochberg method was used to correct for multiple comparisons in five registration methods (AT, DIR, DIR-1, DIR-2, and DIR-3). A level of $\mathrm{P}<0.05$ was considered statistically significant, and all statistical analyses were performed in $\mathrm{R}$ (version 3.6.3).

The resulting transformations' plausibility was assessed 

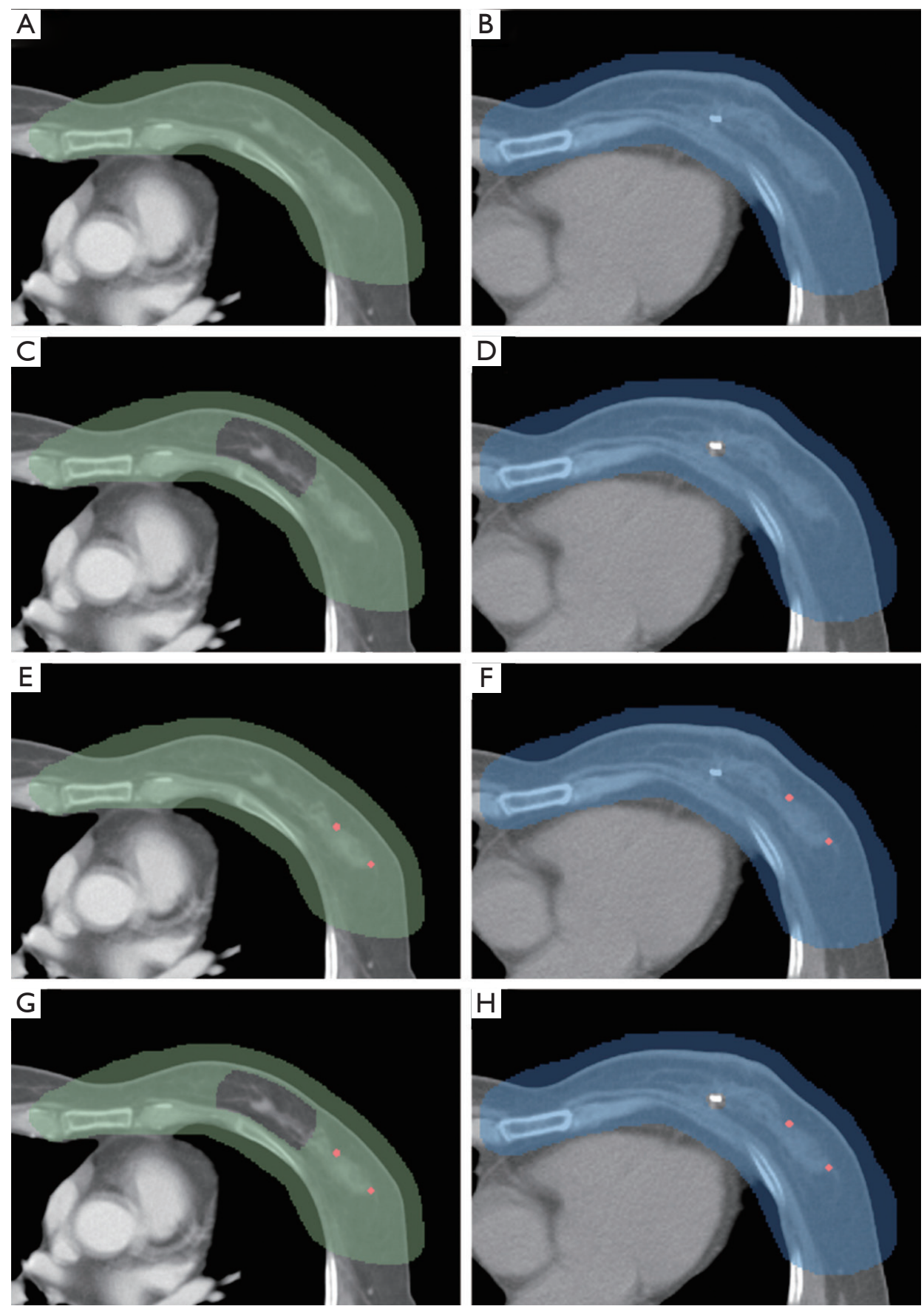

Figure 2 Examples of fixed and moving images in four deformable image registration (DIR) methods. (A) Fixed image of DIR; (B) moving image of DIR; (C) fixed image of DIR-1; (D) moving image of DIR-1; (E) fixed image of DIR-2; (F) moving image of DIR-2; (G) fixed image of DIR-3; (H) moving image of DIR-3. The green label represents the fixed image mask (if tumor expansion is not subtracted, it means no mask) and the blue label represents the moving image mask (if postoperative changes are not subtracted, it means no mask). Point pairs for calculating point metric are represented by red dots. For DIR, the conventional method consisted of the fixed image and the moving image both without point metric and without masking technique. For DIR-1, the first combination consisted of the fixed image and the moving image both without point metric but with masking technique. For DIR-2, the second combination consisted of the fixed image and the moving image both with point metric but without masking technique. For DIR-3, the third combination consisted of the fixed image and the moving image both with point metric and with masking technique. DIR, deformable image registration. 

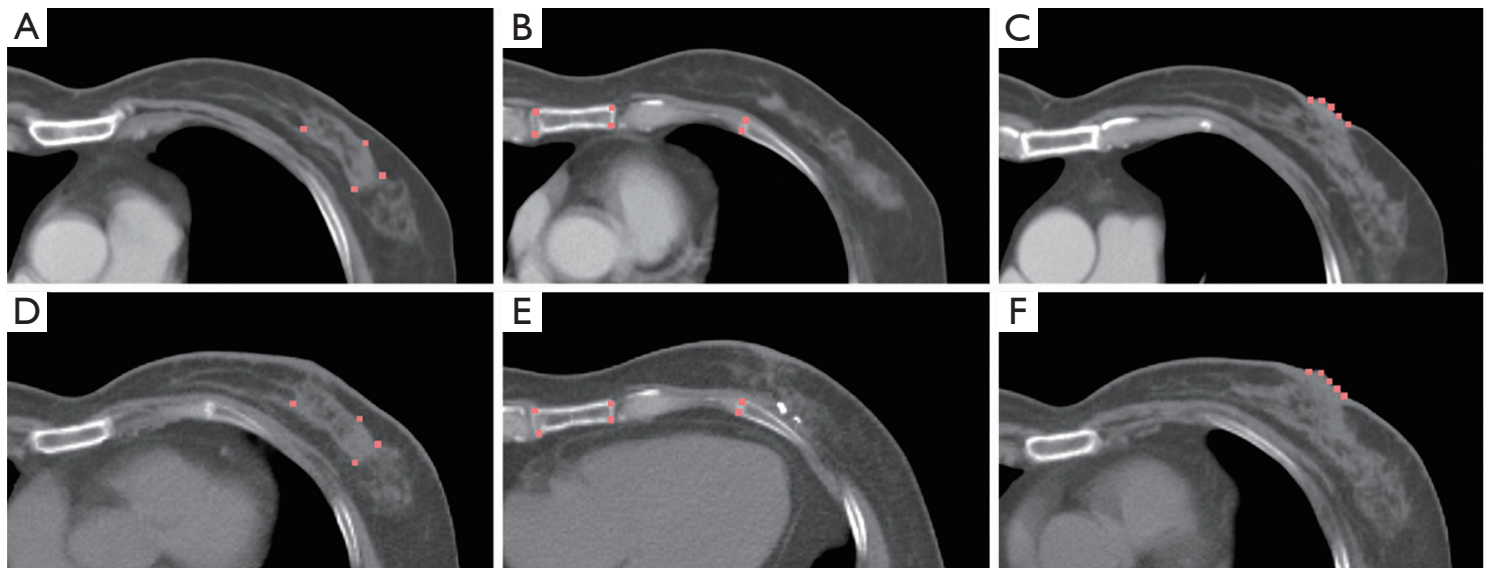

Figure 3 Three categories of point sets chosen for calculating target registration error (TRE). (A) Soft tissues next to the tumor area (fixed image); (B) rigid structures (fixed image); (C) body boundaries (fixed image); (D) soft tissues next to the tumor area (moving image); (E) rigid structures (moving image); (F) body boundaries (moving image). These points are represented by red dots.

by the Jacobian determinant of the displacement vector field (DVF). The Jacobian determinant identifies the local volume change as a result of the registration. Values greater than 1 indicate volume expansion. Values between 0 and 1 indicate volume reduction. Value 1 indicates no change. A value less than or equal to 0 indicates non-physical motion. This quantity can be used to screen the transformation matrix for any ill behaviors. However, positive Jacobians do not automatically guarantee registration accuracy because large local changes in the Jacobian determinant can also indicate a registration error (e.g., voxels in a small $2 \times 2$ region with a Jacobian of 10 while surrounding voxels have a Jacobian of 1).

Visual assessment of medical images is necessary because it is easy to identify improper results based on the physician's clinical experience. The checkerboard image is one of the most commonly-used qualitative visualization tools for detecting registration error. It is particularly effective for identifying mismatches between corresponding structures at high contrast tissue interfaces (16). Besides, image overlay display produces images that are the blended composition of registered images. Fixed and deformed moving images are displayed in opposite colors (red and cyan), and all perfectly aligned structures are displayed in gray. Any deviations are shown in either red or cyan for fixed or deformed moving images, respectively. Each patient CT image was evaluated by two experienced physicians within 4 to 5 minutes.

\section{Results}

The running time of a single test including affine and B-splines based registration averaged 150 seconds. With the application of point metric and masking technique, the running time was 158 seconds. On average, the time taken to place points was 8 minutes, and the time taken to set the masks was 3 minutes.

The statistics of TRE for the five different methods are listed in Table 1. The corrected $\mathrm{P}$ values for multiple comparisons are shown in Figure 4. The initial AT achieved median TREs of 7.38, 5.82, and $8.17 \mathrm{~mm}$ for soft tissue, rigid structure, and boundaries, respectively. The Wilcoxon signed-rank test was performed to compare the AT and other DIR methods. The $\mathrm{P}$ values were less than 0.05 , which indicated significance with the application of DIR methods. Except for soft tissue alignment, there was no statistical difference between AT and DIR methods without point metric (DIR and DIR-1). Specifically, the $\mathrm{P}$ value was 0.29 between AT and DIR, and the P value was 0.19 between AT and DIR-1.

Among the different DIR methods listed in Table 1, the application of point metric (DIR-2 and DIR-3) significantly improved the soft tissue alignment. Specifically, without applying masking technique, TRE decreased from 5.88 to $2.51 \mathrm{~mm}$ (comparison between DIR and DIR-2). With the application of masking technique, TRE decreased from 5.28 to $2.27 \mathrm{~mm}$ (comparison between DIR-1 and DIR-3). In evaluating rigid structures, all DIR methods met 
Table 1 The results of TRE (mm) for AT and DIR methods

\begin{tabular}{|c|c|c|c|c|c|c|c|c|c|}
\hline \multirow{2}{*}{ Methods } & \multicolumn{3}{|c|}{ Boundary } & \multicolumn{3}{|c|}{ Rigid structure } & \multicolumn{3}{|c|}{ Soft tissue } \\
\hline & Median & 25 PCTL & 75 PCTL & Median & 25 PCTL & 75 PCTL & Median & 25 PCTL & 75 PCTL \\
\hline DIR & 3.12 & 2.61 & 6.05 & 2.63 & 1.50 & 3.72 & 5.88 & 3.60 & 6.47 \\
\hline DIR-2 & 2.87 & 2.17 & 3.61 & 2.35 & 1.55 & 3.72 & 2.51 & 1.85 & 2.65 \\
\hline DIR-3 & 3.28 & 2.02 & 4.53 & 2.25 & 1.59 & 3.71 & 2.27 & 1.91 & 2.58 \\
\hline
\end{tabular}

The columns showed the statistics (median, 25 and 75 PCTL values) corresponding to three categories of point sets (boundary, rigid structure, and soft tissue). PCTL, percentile.

Boundary

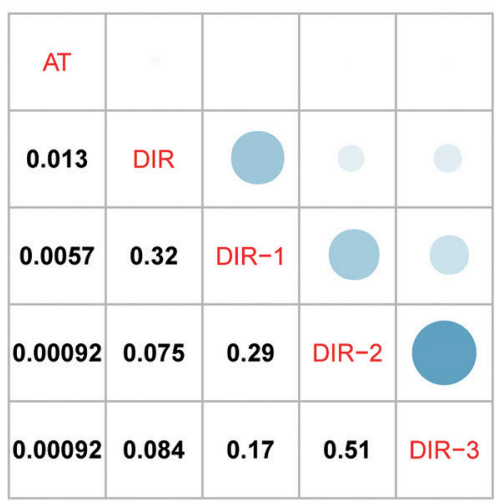

Rigid structure

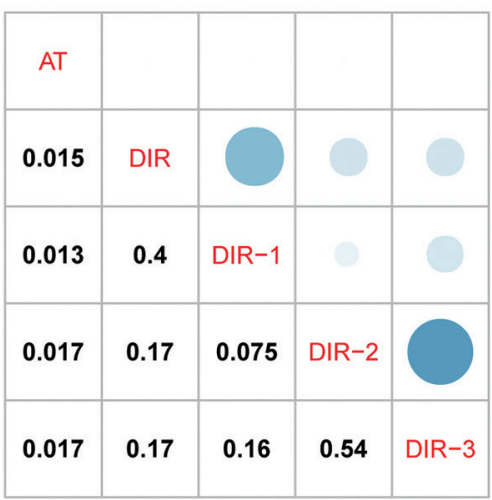

Soft tissue

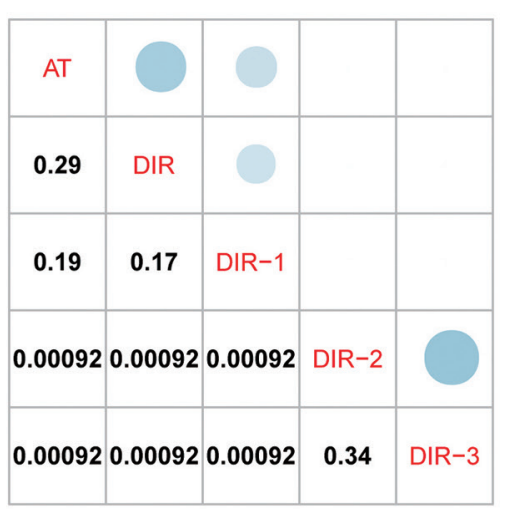

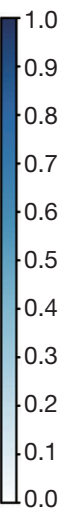

Figure 4 The corrected $\mathrm{P}$ values for multiple comparisons of the five registration methods. A level of $\mathrm{P}<0.05$ was considered statistically significant. For DIR, the conventional method consisted of the fixed image and the moving image both without point metric and without masking technique. For DIR-1, the first combination consisted of the fixed image and the moving image both without point metric but with masking technique. For DIR-2, the second combination consisted of the fixed image and the moving image both with point metric but without masking technique. For DIR-3, the third combination consisted of the fixed image and the moving image both with point metric and with masking technique. AT, affine transformation; DIR, deformable image registration.

the clinical tolerance of 2-3 mm (16). Although there was no statistically significant improvement with the application of masking technique (DIR-1 compared with DIR, DIR3 compared with DIR-2), a slight decrease in registration error was observed. $\mathrm{P}$ values were all above 0.05 within the three different point sets. However, TRE decreased by $4.6 \%$ and $9.9 \%$ on average in rigid structure and soft tissue alignment, respectively. DIR-3 showed higher accuracy in the registration of soft tissue and rigid structures, but less accuracy in the registration of boundaries.

The statistics of the Jacobian determinant for the 4 DIR methods are listed in Table 2. Representative transverse views of the spatial Jacobian map around the tumor areas are shown in Figure 5. The Jacobian determinant of the deformation was positive for all patients. In general, with the application of point metric, the mean values of the Jacobian determinant decreased (DIR-2 compared with DIR, DIR-3 compared with DIR-1). Without application of masking technique (as shown in Figure $5 A, C$ ), a local expansion of volume near the tumor region was observed, which seemed unrealistic from a clinical point of view.

For qualitative visual assessment, the image overlay 
Table 2 The results of Jacobian determinant for four DIR methods

\begin{tabular}{llll}
\hline Methods & Mean \pm SD & Min & Max \\
\hline DIR & $1.00 \pm 0.19$ & 0.54 & 1.63 \\
DIR-1 & $0.99 \pm 0.16$ & 0.55 & 1.52 \\
DIR-2 & $0.94 \pm 0.21$ & 0.43 & 1.61 \\
DIR-3 & $0.93 \pm 0.18$ & 0.47 & 1.51 \\
\hline
\end{tabular}

The columns showed the statistics (mean \pm standard deviation, minimum and maximum values) corresponding to four DIR methods.
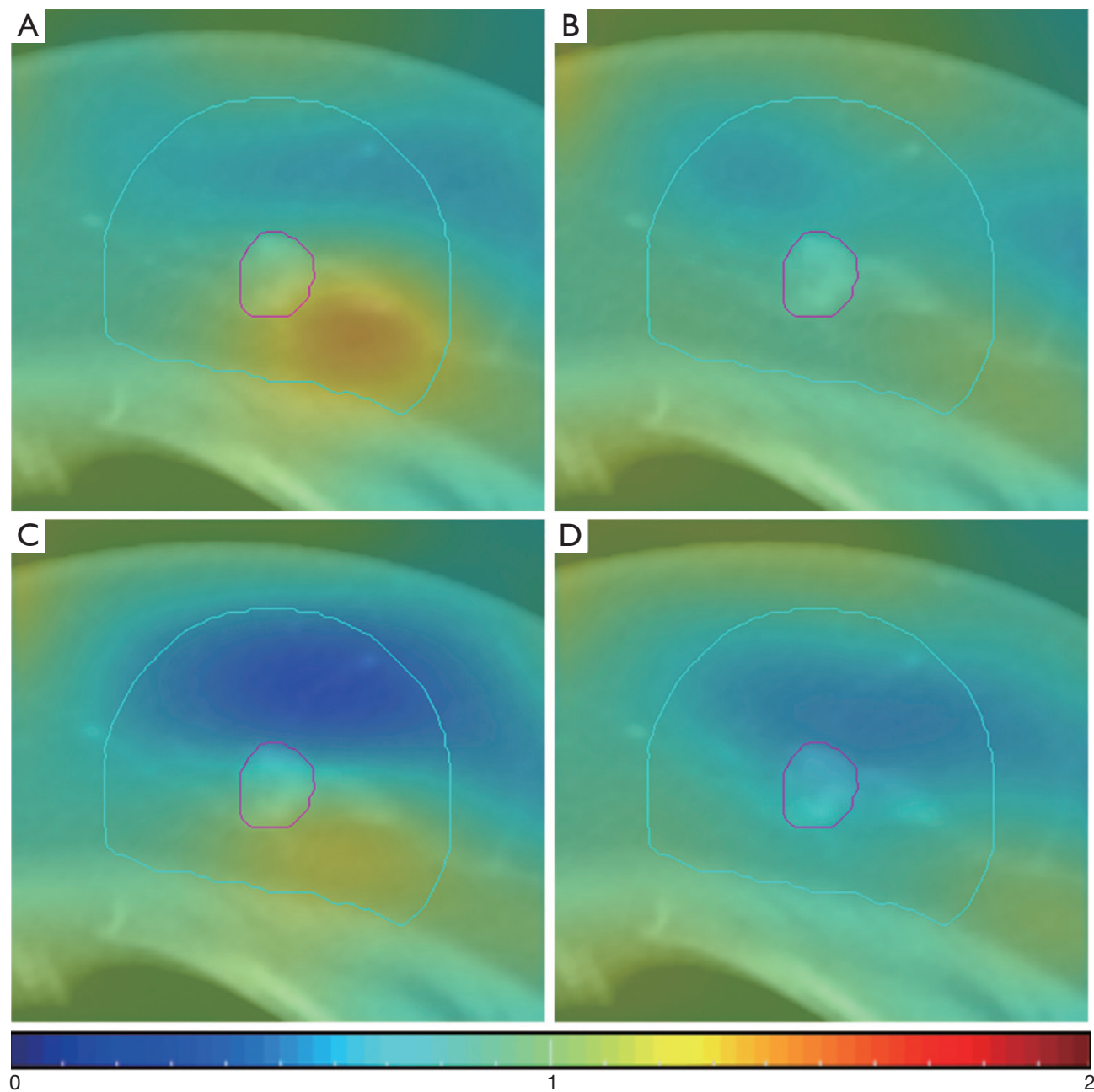

Figure 5 Transverse views of the spatial Jacobian map of the transformation for deformable image registration (DIR) (A), DIR-1 (B), DIR2 (C), DIR-3 (D). Values greater than 1 (red end of spectrum) indicate local volume expansion. Values less than 1 (blue end of spectrum) indicate volume reduction. The pink contour represents the original tumor and the blue contour represents its expansion with a 2 -cm margin. For DIR, the conventional method consisted of the fixed image and the moving image both without point metric and without masking technique. For DIR-1, the first combination consisted of the fixed image and the moving image both without point metric but with masking technique. For DIR-2, the second combination consisted of the fixed image and the moving image both with point metric but without masking technique. For DIR-3, the third combination consisted of the fixed image and the moving image both with point metric and with masking technique. 
displays and the checkerboard images between the fixed and the transformed moving images are shown in Figure 6. Visual assessment of DIR methods was better than that of AT, which was consistent with the TRE metric's evaluation results. Further improvement of soft tissue alignment was observed in the methods with point metric (as shown in Figure $6 G, H, I, 7)$ since the blended displays were almost in gray. However, there was no significant difference between DIR-2 and DIR-3.

\section{Discussion}

The alignment of anatomical landmarks is a direct way to evaluate the accuracy of registration. In this study, the landmarks in soft tissue were within $5 \mathrm{~cm}$ away from the tumor region, which better represents soft tissue movement close to the tumor. Furthermore, the soft tissue points can be more reliable than the other two categories (rigid structures and body boundaries) because they are the closest to the tumor region.

The introduction of point metric is critical for DIR methods. As shown in Table 1, without the application of point metric (DIR and DIR-1), the soft tissue alignment was poor and did not meet the clinical tolerance. With the masking technique's application only (DIR-1), the improvement of soft tissue alignment was limited. This might have been caused by the intensity changes of noncorresponding regions, as well as inaccurate masking area. With the application of point metric and masking technique (DIR-3), higher registration accuracy in soft tissue and rigid structures was obtained. However, the registration accuracy in the boundaries was poorer. This is partly due to surgery's unexpected effect, where some patients may undergo extensive remodeling of the nipples (presented as the boundaries).

The spatial Jacobian image can help to identify certain unrealistic results according to the clinical scenario. Our hospital's surgical regimen did not involve oncoplastic surgery, where the breast tissue is remodeled extensively $(10,11)$. Thus, the Jacobian determinant would not deviate too far from 1, especially near the excision area. The mean values of the Jacobian determinants were smaller with the application of point metric (DIR-2 compared with DIR, DIR-3 compared with DIR-1), which is more consistent with the volume reduction caused by surgery. Without applying masking technique (as shown in Figure $5 A, C$ ), there is a local expansion of volume near the $2 \mathrm{~cm}$ expansion of the tumor. It seems unrealistic because the $2 \mathrm{~cm}$ expansion is approximate to the real surgical margin of the tumor. Thus, the masking technique is recommended for more realistic modeling of the deformation on the expansion of the tumor region.

For qualitative visualization, the region next to the tumor can be more reliable in assessing accuracy. Therefore, in our study, unaffected soft tissues were inspected more carefully. The method with point metric achieved better matching for soft tissue, regardless of applying masking technique.

Contour-based DIR or hybrid DIR methods have been applied in various sites. Incorporating contour information with intensity-based registration can achieve better registration accuracy. However, in our study, the ROIs that could be used as constraints were almost unavailable, which precludes the application of the contour-based or hybrid DIR methods. Our method had significant advantages in terms of performance compared with other open source DIR frameworks (30). Based on preliminary results, our work achieved comparable results to commercial image registration software $(31,32)$ in terms of the TRE tolerance, according to the AAPM TG-132. The proposed method could be applied to other sites, especially for those noncorresponding regions composed of low contrast tissue.

DIR methods that employ both image intensity and landmarks hold great promise for medical image registration applications (33). However, these methods lack robustness for clinical applications since the landmarks are less available in routine practice (34). Moreover, there exists the possibility of inter-observer disagreement in identifying landmarks, especially in low contrast regions, which has also been investigated by some researchers (35). As for the application of masking technique for diagnostic CT, $2 \mathrm{~cm}$ is generally recognized as the safety margin in breastconserving surgery. However, the asymmetric excisional margin varies from person to person in clinical practice. Thus, a more appropriate range of masking should be considered in order to achieve more reasonable delineation of CTV.

There are several limitations of this study. First, the image data sets were limited, as the patients were required to undergo diagnostic CT as a preoperative work-up. More data should be collected in the future. Second, the evaluation was susceptible to subjectivity of human operator. More physicians should, therefore, be recruited for evaluation in the future. Third, the CT image's slice resolution in this study was limited to $5 \mathrm{~mm}$ due to clinical protocol. A finer thickness $(<3 \mathrm{~mm})$ will result in better registration accuracy, where the improvement is marginal 
A

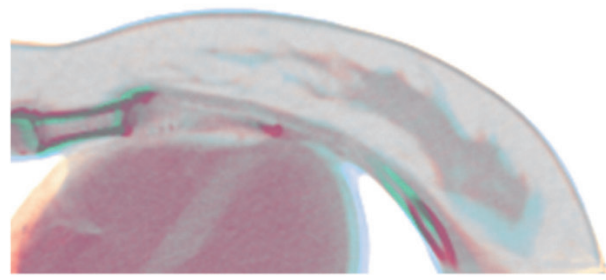

C

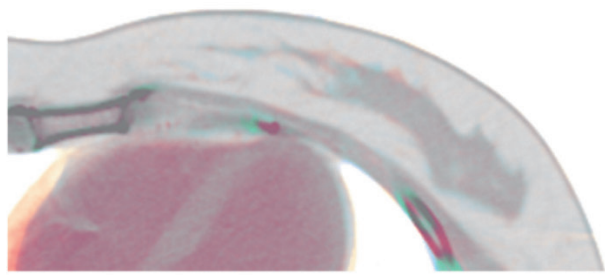

$\mathrm{E}$

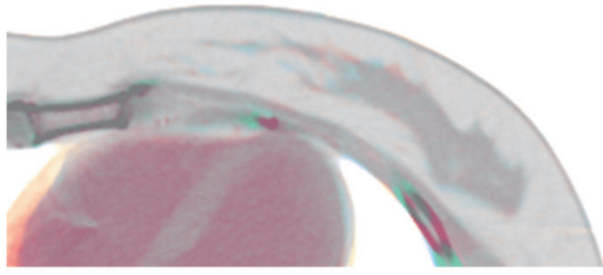

G

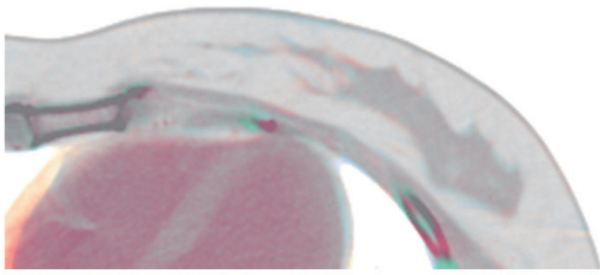

I

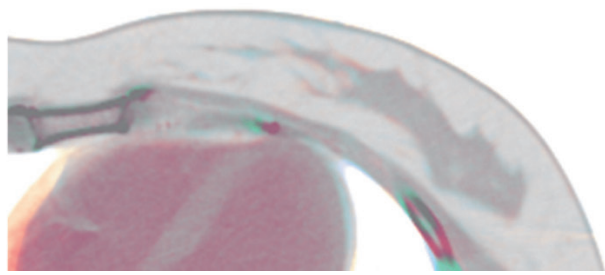

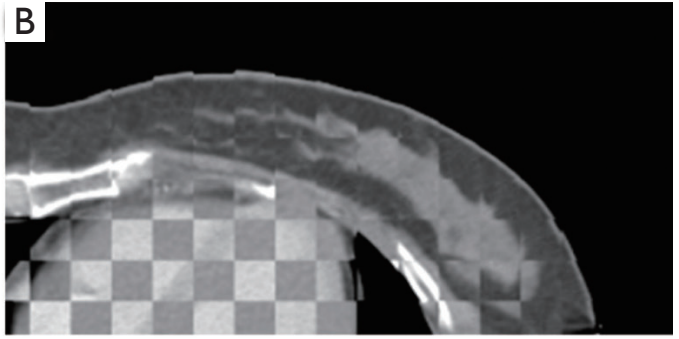

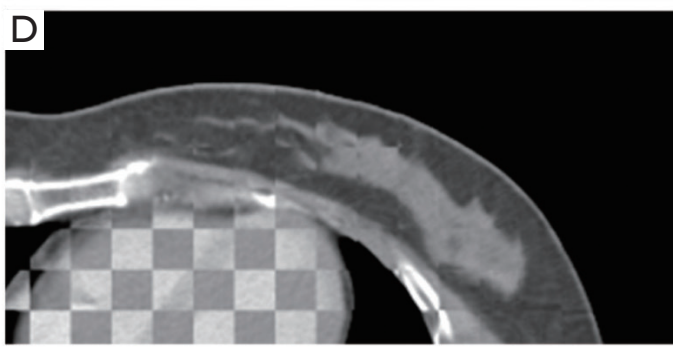

F

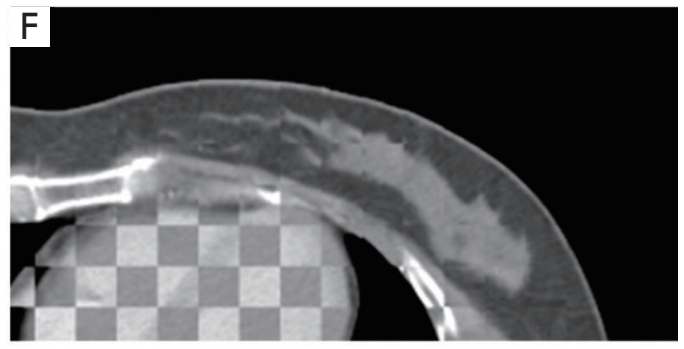

$\mathrm{H}$
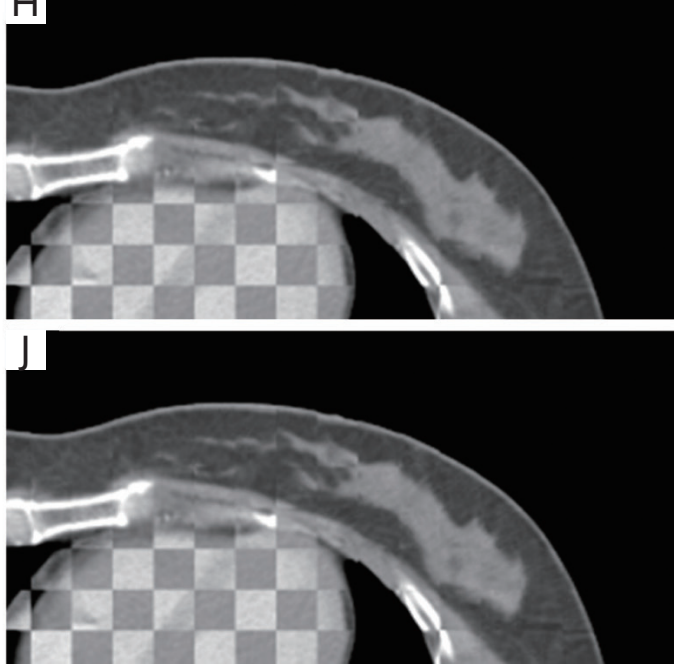

Figure 6 Image overlay displays and checkerboard images for visual assessment. (A) Image overlay display of the affine transformation (AT); (B) checkerboard image of AT; (C) image overlay display of deformable image registration (DIR); (D) checkerboard image of DIR; (E) image overlay display of DIR-1; (F) checkerboard image of DIR-1; (G) image overlay display of DIR-2; (H) checkerboard image of DIR-2; (I) image overlay display of DIR-3; (J) checkerboard image of DIR-3. For DIR, the conventional method consisted of the fixed image and the moving image both without point metric and without masking technique. For DIR-1, the first combination consisted of the fixed image and the moving image both without point metric but with masking technique. For DIR-2, the second combination consisted of the fixed image and the moving image both with point metric but without masking technique. For DIR-3, the third combination consisted of the fixed image and the moving image both with point metric and with masking technique. 
with $1 \mathrm{~mm}$ according to previous reports (36). Therefore, $3 \mathrm{~mm}$ is preferred for clinical use and will be adopted in future studies.

In this study, the corresponding point sets were recognized manually by experienced physicians. This procedure can be greatly improved by automatic detection algorithms and deep learning methods in terms of efficiency and precision. Furthermore, additional feature extraction and other intensity-based registration methods should be explored. Since the points used for regularization in the DIR method are extracted from unaffected glandular tissue, the gland's entire contour could also be used as an additional feature. It may also serve as an ROI for quantitative assessment, which is supplementary to current evaluation methods.

\section{Conclusions}

The introduction of point metric and masking technique to the intensity-based B-splines DIR method is effective for registering CT images before and after breast-conserving surgery. The influence of large deformations and noncorrespondence on the DIR method's registration accuracy can be reduced to a certain degree. This method provides a feasible way for target volume definition in postoperative breast cancer radiotherapy treatment planning.

\section{Acknowledgments}

Funding: This work is partially supported by the National Natural Science Foundation of China (11875320) and Beijing Hope Run Special Fund of Cancer Foundation of China (LC2018A08).

\section{Footnote}

Conflicts of Interest: All authors have completed the ICMJE uniform disclosure form (available at http://dx.doi. org/10.21037/qims-20-705). The authors report grants from the National Natural Science Foundation of China and grants from Beijing Hope Run Special Fund of Cancer Foundation of China during the conduct of the study.

Ethical Statement: This study was approved by the institutional Ethics Committee of Cancer Hospital, Chinese Academy of Medical Sciences and Peking Union Medical College. Informed consent was waived in this retrospective study.
Open Access Statement: This is an Open Access article distributed in accordance with the Creative Commons Attribution-NonCommercial-NoDerivs 4.0 International License (CC BY-NC-ND 4.0), which permits the noncommercial replication and distribution of the article with the strict proviso that no changes or edits are made and the original work is properly cited (including links to both the formal publication through the relevant DOI and the license). See: https://creativecommons.org/licenses/by-nc-nd/4.0/.

\section{References}

1. Bray F, Ferlay J, Soerjomataram I, Siegel RL, Torre LA, Jemal A. Global cancer statistics 2018: GLOBOCAN estimates of incidence and mortality worldwide for 36 cancers in 185 countries. CA Cancer J Clin 2018;68:394-424.

2. Litière $S$, Werutsky G, Fentiman IS, Rutgers E, Christiaens MR, Van Limbergen E, Baaijens MH, Bogaerts J, Bartelink H. Breast conserving therapy versus mastectomy for stage I-II breast cancer: 20 year followup of the EORTC 10801 phase 3 randomised trial. Lancet Oncol 2012;13:412-9.

3. Bartelink H, Horiot JC, Poortmans PM, Struikmans H, Van den Bogaert W, Fourquet A, Jager JJ, Hoogenraad WJ, Oei SB, Warlam-Rodenhuis CC, Pierart M, Collette L. Impact of a higher radiation dose on local control and survival in breast-conserving therapy of early breast cancer: 10-year results of the randomized boost versus no boost EORTC 22881-10882 trial. J Clin Oncol 2007;25:3259-65.

4. Livi L, Meattini I, Marrazzo L, Simontacchi G, Pallotta S, Saieva C, Paiar F, Scotti V, De Luca Cardillo C, Bastiani P, Orzalesi L, Casella D, Sanchez L, Nori J, Fambrini M, Bianchi S. Accelerated partial breast irradiation using intensity-modulated radiotherapy versus whole breast irradiation: 5-year survival analysis of a phase 3 randomised controlled trial. Eur J Cancer 2015;51:451-63.

5. van Mourik AM, Elkhuizen PH, Minkema D, Duppen JC, van Vliet-Vroegindeweij C. Multiinstitutional study on target volume delineation variation in breast radiotherapy in the presence of guidelines. Radiother Oncol 2010;94:286-91.

6. Guo Y, Sivaramakrishna R, Lu CC, Suri JS, Laxminarayan S. Breast image registration techniques: a survey. Med Biol Eng Comput 2006;44:15-26.

7. Kirova YM, Fournier-Bidoz N, Servois V, Laki F, Pollet GA, Salmon R, Thomas A, Dendale R, Bollet MA, 
Campana F, Fourquet A. How to boost the breast tumor bed? A multidisciplinary approach in eight steps. Int J Radiat Oncol Biol Phys 2008;72:494-500.

8. Kirova YM, Castro Pena P, Hijal T, Fournier-Bidoz N, Laki F, Sigal-Zafrani B, Dendale R, Bollet MA, Campana F, Fourquet A. Improving the definition of tumor bed boost with the use of surgical clips and image registration in breast cancer patients. Int J Radiat Oncol Biol Phys 2010;78:1352-5.

9. Boersma LJ, Janssen T, Elkhuizen PH, Poortmans P, van der Sangen M, Scholten AN, Hanbeukers B, Duppen JC, Hurkmans C, van Vliet C. Reducing interobserver variation of boost-CTV delineation in breast conserving radiation therapy using a pre-operative CT and delineation guidelines. Radiother Oncol 2012;103:178-82.

10. Wodzinski M, Skalski A, Ciepiela I, Kuszewski T, Kedzierawski P, Gajda J. Improving oncoplastic breast tumor bed localization for radiotherapy planning using image registration algorithms. Phys Med Biol 2018;63:035024.

11. Kirova YM, Servois V, Reyal F, Peurien D, Fourquet A, Fournier-Bidoz N. Use of deformable image fusion to allow better definition of tumor bed boost volume after oncoplastic breast surgery. Surg Oncol 2011;20:e123-5.

12. Yu T, Li JB, Wang W, Xu M, Zhang YJ, Shao Q, Liu XJ, $\mathrm{Xu} \mathrm{L}$. A comparative study based on deformable image registration of the target volumes for external-beam partial breast irradiation defined using preoperative prone magnetic resonance imaging and postoperative prone computed tomography imaging. Radiat Oncol 2019;14:38.

13. Cho O, Chun M, Oh YT, Kim MH, Park HJ, Heo JS, Noh OK. Can initial diagnostic PET-CT aid to localize tumor bed in breast cancer radiotherapy: feasibility study using deformable image registration. Radiat Oncol 2013;8:163.

14. Ruhaak J, Polzin T, Heldmann S, Simpson IJA, Handels H, Modersitzki J, Heinrich MP. Estimation of Large Motion in Lung CT by Integrating Regularized Keypoint Correspondences into Dense Deformable Registration. IEEE Trans Med Imaging 2017;36:1746-57.

15. Sotiras A, Davatzikos C, Paragios N. Deformable medical image registration: a survey. IEEE Trans Med Imaging 2013;32:1153-90.

16. Brock KK, Mutic S, McNutt TR, Li H, Kessler ML. Use of image registration and fusion algorithms and techniques in radiotherapy: Report of the AAPM Radiation Therapy Committee Task Group No. 132. Med Phys 2017;44:e43-76.
17. Zhong H, Kim J, Gordon JJ, Brown SL, Movsas B, Chetty IJ. editors. Morphological Analysis of Tumor Regression and Its Impact on Deformable Image Registration for Adaptive Radiotherapy of Lung Cancer Patients. Cham: Springer International Publishing, 2015.

18. Chen Y, Yin FF, Zhang Y, Zhang Y, Ren L. Low dose conebeam computed tomography reconstruction via hybrid prior contour based total variation regularization (hybrid-PCTV). Quant Imaging Med Surg 2019;9:1214-28.

19. Major T, Gutierrez C, Guix B, van Limbergen E, Strnad V, Polgar C. Recommendations from GEC ESTRO Breast Cancer Working Group (II): Target definition and target delineation for accelerated or boost partial breast irradiation using multicatheter interstitial brachytherapy after breast conserving open cavity surgery. Radiother Oncol 2016;118:199-204.

20. Hilts M, Batchelar D, Rose J, Crook J. Deformable image registration for defining the postimplant seroma in permanent breast seed implant brachytherapy. Brachytherapy 2015;14:409-18.

21. Loi G, Fusella M, Lanzi E, Cagni E, Garibaldi C, Iacoviello G, Lucio F, Menghi E, Miceli R, Orlandini LC, Roggio A, Rosica F, Stasi M, Strigari L, Strolin S, Fiandra C. Performance of commercially available deformable image registration platforms for contour propagation using patient-based computational phantoms: A multiinstitutional study. Med Phys 2018;45:748-57.

22. Wodzinski M, Skalski A, Ciepiela I, Kuszewski T, Kedzierawski P. Application of Demons Image Registration Algorithms in Resected Breast Cancer Lodge Localization. 2017 Signal Processing: Algorithms, Architectures, Arrangements, and Applications (SPA). 20-22 Sep. 2017; Poznan, Poland. IEEE, 2017:400-5.

23. Wodzinski M, Skalski A, Kedzierawski P, Kuszewski T. Application of B-Splines FFD Image Registration in Breast Cancer Radiotherapy Planning. 2017 International Conference on Systems, Signals and Image Processing (IWSSIP). 22-24 May 2017; Poznan, Poland. IEEE, 2017.

24. Wodzinski M, Skalski A, Kedzierawski P, Kuszewski T, Ciepiela I. Usage of ICP Algorithm for Initial Alignment in B-Splines FFD Image Registration in Breast Cancer Radiotherapy Planning. In: Augustyniak P, Maniewski $\mathrm{R}$, Tadeusiewicz R. editors. Recent Developments and Achievements in Biocybernetics and Biomedical Engineering. Polish Conference on Biocybernetics and Biomedical Engineering 2017. Advances in Intelligent Systems and Computing, vol 647. Springer, Cham, 2018:143-52. 
25. Rueckert D, Sonoda LI, Hayes C, Hill DL, Leach MO, Hawkes DJ. Nonrigid registration using free-form deformations: application to breast MR images. IEEE Trans Med Imaging 1999;18:712-21.

26. Baiker M, Staring M, Lowik CW, Reiber JH, Lelieveldt BP. Automated registration of whole-body follow-up MicroCT data of mice. Med Image Comput Comput Assist Interv 2011;14:516-23.

27. Klein S, Staring M, Murphy K, Viergever MA, Pluim JP. elastix: a toolbox for intensity-based medical image registration. IEEE Trans Med Imaging 2010;29:196-205.

28. Shamonin DP, Bron EE, Lelieveldt BP, Smits M, Klein S, Staring M. Fast parallel image registration on CPU and GPU for diagnostic classification of Alzheimer's disease. Front Neuroinform 2014;7:50.

29. Heinrich MP, Jenkinson M, Bhushan M, Matin T, Gleeson FV, Brady SM, Schnabel JA. MIND: modality independent neighbourhood descriptor for multi-modal deformable registration. Med Image Anal 2012;16:1423-35.

30. Mogadas N, Sothmann T, Knopp T, Gauer T, Petersen C, Werner R. Influence of deformable image registration on 4D dose simulation for extracranial SBRT: A multi-registration framework study. Radiother Oncol 2018;127:225-32.

Cite this article as: Xie X, Song Y, Ye F, Yan H, Wang S, Zhao X, Dai J. Improving deformable image registration with point metric and masking technique for postoperative breast cancer radiotherapy. Quant Imaging Med Surg 2021;11(4):1196-1208. doi: 10.21037/qims-20-705
31. Motegi K, Tachibana H, Motegi A, Hotta K, Baba H, Akimoto T. Usefulness of hybrid deformable image registration algorithms in prostate radiation therapy. J Appl Clin Med Phys 2019;20:229-36.

32. Kubota Y, Okamoto M, Li Y, Shiba S, Okazaki S, Komatsu S, Sakai M, Kubo N, Ohno T, Nakano T. Evaluation of Intensity- and Contour-Based Deformable Image Registration Accuracy in Pancreatic Cancer Patients. Cancers (Basel) 2019;11:1447.

33. Yin Y, Hoffman EA, Ding K, Reinhardt JM, Lin CL. A cubic B-spline-based hybrid registration of lung CT images for a dynamic airway geometric model with large deformation. Phys Med Biol 2011;56:203-18.

34. Liang X, Yin FF, Wang C, Cai J. A robust deformable image registration enhancement method based on radial basis function. Quant Imaging Med Surg 2019;9:1315-25.

35. Luu HM, Klink C, Niessen W, Moelker A, van Walsum $\mathrm{T}$. An automatic registration method for pre- and postinterventional CT images for assessing treatment success in liver RFA treatment. Med Phys 2015;42:5559-67.

36. Sarudis S, Karlsson A, Bibac D, Nyman J, Bäck A. Evaluation of deformable image registration accuracy for CT images of the thorax region. Phys Med 2019;57:191-9. 


\section{Supplementary}

\section{Appendix 1}

In general, the registration problem is formulated as an optimization problem which maximizes a cost function $C\left(T ; I_{F}, I_{M}\right)$ with respect to the transform $T$. The transform $T$ is obtained by optimizing the control point location in a manner which maximizes the similarity metric between the fixed image $\left(I_{F}\right)$ and the moving image $\left(I_{M}\right)$,

$$
\begin{aligned}
& {[1] \hat{\mathrm{T}}=\arg \max _{\mathrm{T}} \mathrm{C}\left(\mathrm{T} ; \mathrm{I}_{\mathrm{F}}, \mathrm{I}_{\mathrm{M}}\right)} \\
& \text { with } \\
& \mathrm{C}\left(\mathrm{T} ; \mathrm{I}_{\mathrm{F}}, \mathrm{I}_{\mathrm{M}}\right)=-\mathrm{S}\left(\mathrm{T} ; \mathrm{I}_{\mathrm{F}}, \mathrm{I}_{\mathrm{M}}\right)+\alpha \mathrm{P}(\mathrm{T})
\end{aligned}
$$

where $P(T)$ is a regularization term which constrains nonrigid deformation and $\alpha$ is a weighting factor which balances the similarity metric $S\left(T ; I_{F}, I_{M}\right)$ and the regularization term $P(T)$. We model the non-rigid transform $T$ with a B-Splines based deformation field, where parameter $\mu$ models the transformation $T$. Finding the optimal transformation $\hat{T}$ therefore is an optimization problem, determining the parameter $\mu$ that maximizes the cost function $C\left(\mu ; I_{F}, I_{M}\right)$,

$$
\widehat{\mathrm{T}_{\mu}}=\arg \max _{\mathrm{T}} \mathrm{C}\left(\mathrm{T}_{\mu} ; \mathrm{I}_{\mathrm{F}}, \mathrm{I}_{\mathrm{M}}\right)
$$

$$
\begin{aligned}
& \text { or } \\
& \mu=\arg \max _{\mathrm{T}} \mathrm{C}\left(\mu ; \mathrm{I}_{\mathrm{F}}, \mathrm{I}_{\mathrm{M}}\right)
\end{aligned}
$$

In every iteration $k$, the current parameter $\mu_{k}$ is updated by adding a small step in direction of the derivative of the cost function $\partial C / \partial \mu$,

$$
\mu_{\mathrm{k}+1}=\mu_{\mathrm{k}}-\mathrm{a}_{\mathrm{k}} \frac{\partial \mathrm{C}}{\partial \mu}
$$

where $\alpha_{k}>0$ is the size of the step which changes in every iteration. Klein et al. (37) proved that using a decay of $\alpha_{k}$ according to $a_{k}=a /(k+A)^{\gamma}$, where $\alpha>0, \mathrm{~A} \geq 1$, and $0 \leq y \leq 1$ are user-predefined constants, the convergence rate significantly reduces computation time without affecting the final result. Based on this result, we used the stochastic gradient descent in our study.

\section{References}

37. Klein S, Pluim JPW, Staring M, Viergever MA. Adaptive Stochastic Gradient Descent Optimisation for Image Registration. Int J Comput Vis 2008;81:227. 